\title{
Supporting Information: Stochastic Mechanisms of Cell-Size Regulation in Bacteria
}

September 24, 2020

In this Supporting Information we provide the details of calculations for the equations utilized in the main text.

\section{Calculation of the variance in the added cell length}

As it is shown in the main text, the probability that the cell will divide after increasing its size by exactly $n$ units can be written as $Q_{n}=p(1-p)^{n}$. Hence, the second moment of added volume reads:

$$
<l^{2}>=\sum_{n=0}^{\infty} n^{2} Q_{n}=\sum_{n=0}^{\infty} n^{2} p(1-p)^{n}=\frac{2-3 p+p^{2}}{p^{2}}
$$

Thus variance is given by:

$$
\sigma=\sqrt{<l^{2}>-<l>^{2}}=\sqrt{\frac{1-p}{p^{2}}} .
$$

For convenience we also calculate the normalized variance, which is a dimensionless quantity:

$$
\bar{\sigma}=\frac{\sqrt{<l^{2}>-<l>^{2}}}{<l>}=\frac{\sqrt{\frac{1-p}{p^{2}}}}{\frac{1-p}{p}}=\frac{1}{\sqrt{1-p}}=\sqrt{\frac{1+\lambda / k}{\lambda / k}} .
$$

\section{Calculation of the inter-division times}

Following the discussion in the main text, we present the detailed calculation of the average time before cell division using the mean first passage method. We define $F_{m}(t)$ as the probability to dissociate from the state $\left(n_{0}+l\right)$ at time $t$ if at $t=0$ the system started in the state $m$ (see Fig. 1). The dynamics in the system can be described by a set of backward master equations:

$$
\frac{d F_{m}(t)}{d t}=\lambda m F_{m+1}(t)-(\lambda+k) m F_{m}(t)
$$




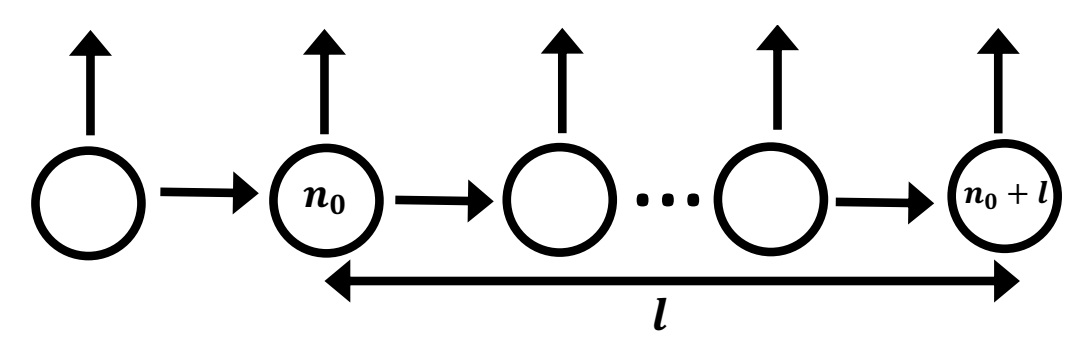

Figure 1: Modified schematic representation of the discrete-state stochastic model to describe the first passage approach.

$$
\frac{d F_{n_{0}+l}(t)}{d t}=k\left(n_{0}+l\right) F_{d}(t)-(\lambda+k)\left(n_{0}+l\right) F_{n_{0}+l}(t)
$$

where, $F_{d}(t)=\delta(t)$ is the state after division at $\left(n_{0}+l\right)$. Performing Laplace transform, the equations $\mathrm{S} 4$ and $\mathrm{S} 5$ can be written as:

$$
\begin{gathered}
{[s+(\lambda+k) m] \widetilde{F}_{m}=\lambda m \widetilde{F}_{m+1}} \\
{\left[s+(\lambda+k)\left(n_{0}+l\right)\right] \widetilde{F}_{n_{0}+l}=k\left(n_{0}+l\right)}
\end{gathered}
$$

The equation $\mathrm{S} 7$ can be further simplified as:

$$
\widetilde{F}_{n_{0}+l}=\frac{k}{\lambda} \cdot \frac{\lambda}{\left(\frac{s}{n_{0}+l}+\lambda+k\right)}
$$

Using equation $\mathrm{S} 6$ we get;

$$
\widetilde{F}_{n_{0}+l-i}=\frac{k}{\lambda} \cdot \frac{\lambda^{i+1}}{\prod_{j=0}^{i}\left(\frac{s}{n_{0}+l-j}+\lambda+k\right)}
$$

where $i=0, \ldots, l$. For $l=m$ we obtain:

$$
\widetilde{F}_{n_{0}}=\frac{k}{\lambda} \cdot \frac{\lambda^{l+1}}{\prod_{j=0}^{l}\left(\frac{s}{n_{0}+l-j}+\lambda+k\right)}
$$

From the first passage distribution, we can obtain the splitting probability, $\pi_{n_{0}}$ of dividing at the state $n_{0}+l$.

$$
\pi_{n_{0}}=\widetilde{F}_{n_{0}}(s=0)=\frac{k \lambda^{l+1}}{\lambda(\lambda+k)^{l+1}}=p(1-p)^{l}
$$

Moreover, this method allows us to obtain the mean-first passage time, $T_{n_{0}}$ :

$$
T_{n_{0}}=-\frac{\left.\frac{\partial \widetilde{F}_{n_{0}}(s)}{\partial s}\right|_{s=0}}{\widetilde{F}_{n_{0}}(s=0)}=\frac{1}{k+\lambda} \sum_{j=0}^{l} \frac{1}{n_{0}+l-j}
$$

It can be shown that $T_{n_{0}}$ can be simplified as:

$$
T_{n_{0}}=\frac{1}{k+\lambda} \sum_{j=0}^{l} \frac{1}{n_{0}+j}
$$


In Fig.2 we compared this result with Monte-Carlo simulations. Similarly we can obtain the second moment of the inter-division times:

$$
T_{n_{0}}^{2}=\frac{\left.\frac{\partial^{2} \widetilde{F}_{n_{0}}(s)}{\partial s^{2}}\right|_{s=0}}{\widetilde{F}_{n_{0}}(s=0)}
$$

After some algebra we obtain:

$$
T_{n_{0}}^{2}=\frac{1}{(k+\lambda)^{2}} \sum_{j=0}^{l} \frac{1}{\left(n_{0}+l-j\right)^{2}}+\frac{1}{(k+\lambda)^{2}}\left(\sum_{j=0}^{l} \frac{1}{\left(n_{0}+l-j\right)}\right)^{2}
$$

Consequently we can obtain the variance of inter-division times:

$$
\delta T_{n_{0}}=\sqrt{T_{n_{0}}^{2}-\left(T_{n_{0}}\right)^{2}}=\frac{1}{(k+\lambda)} \sqrt{\sum_{j=0}^{l} \frac{1}{\left(n_{0}+l-j\right)^{2}}}
$$

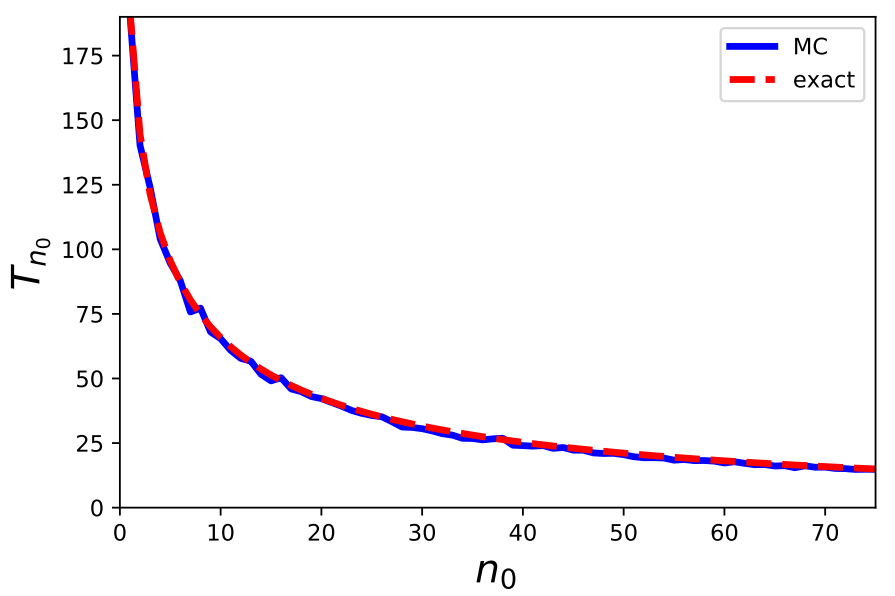

Figure 2: Comparison of simulation results and exact solution for interdivision $T_{n 0}$ versus $n_{0}$.

\section{Propagator method}

In this section we calculate the inter-division times and its variance using propagator method. To do so, we define $G\left(n, t \mid n_{0}\right)$ as the probability density of finding the system at state $n$ at time $t$ conditional on the system being at $n_{0}$ at $t=0$. This probability density, otherwise known as propagator, is governed following master equation:

$$
\frac{d G\left(n, t \mid n_{0}\right)}{d t}=-(k+\lambda) n G\left(n, t \mid n_{0}\right)+\lambda(n-1) G\left(n-1, t \mid n_{0}\right)\left(1-1 \delta_{n, n_{0}}\right)
$$


where $n_{0} \leq n \leq l$, and initial condition $G\left(n, t=0 \mid n_{0}\right)=\delta_{n, n_{0}}$. It is convenient to solve this master equation using Laplace transformation:

$$
\widetilde{G_{n}}=\widetilde{G}\left(n, s \mid n_{0}\right) \equiv \int_{0}^{\infty} e^{-s t} G\left(n, t \mid n_{0}\right) d t
$$

Performing Laplace transform over equation S17 yields:

$$
s \widetilde{G_{n}}-\delta_{n, n_{0}}=-(k+\lambda) n \widetilde{G_{n}}+\lambda(n-1) \widetilde{G_{n-1}}\left(1-\delta_{n, n_{0}}\right)
$$

We define $k+\lambda=J$. Thus

$$
(s+n J) \widetilde{G_{n}}-\lambda(n-1) \widetilde{G_{n-1}}\left(1-\delta_{n, n_{0}}\right)=\delta_{n, n_{0}}
$$

For $n=n_{0}$ we obtain:

$$
\widetilde{G_{n_{0}}}=\frac{1}{s+J n_{0}}
$$

For $n \neq n_{0}$ we obaitn:

$$
\widetilde{G_{n}}=\frac{\lambda(n-1)}{s+n J} \widetilde{G_{n-1}}
$$

Combining eqns S21 and S22 we obtain:

$$
\widetilde{G_{n_{0}+m}}=\frac{\lambda^{m} \prod_{i=0}^{m-1}\left(n_{0}+i\right)}{\prod_{i=0}^{m}\left(s+J\left(n_{0}+i\right)\right)}
$$

To proceed further we define the probability flux $\psi_{n_{0}+m}(t)$ entering state $n_{0}+m$ at time $t$ :

$$
\psi_{n_{0}+m}(t)=\lambda\left(n_{0}+m-1\right) G\left(n_{0}+m-1, t \mid n_{0}\right)
$$

Performing Laplace transformation yields :

$$
\widetilde{\psi}_{n_{0}+m}(s)=\lambda\left(n_{0}+m-1\right) \widetilde{G}\left(n_{0}+m-1, s \mid n_{0}\right)
$$

Thus the probability density of the first-passage time from $n_{0}$ to $n_{0}+m$ denoted by $\phi_{n_{0}+m}(t)$, is given by:

$$
\phi_{n_{0}+m}(t)=\frac{1}{\int_{0}^{\infty} \psi_{n_{0}+m}(t) d t} \psi_{n_{0}+m}(t)
$$

Performing Laplace transformation yields:

$$
\widetilde{\phi}_{n_{0}+m}(s)=\frac{1}{\widetilde{\psi}_{n_{0}+m}(s=0)} \widetilde{\psi}_{n_{0}+m}(s)
$$

Combing equations S25 and S27 yields:

$$
\widetilde{\phi}_{n_{0}+m}(s)=\frac{\widetilde{G}\left(n_{0}+m-1, s \mid n_{0}\right)}{\widetilde{G}\left(n_{0}+m-1, s=0 \mid n_{0}\right)}
$$


Using eqn S23 we obtain:

$$
\widetilde{\phi}_{n_{0}+m}(s)=\frac{J^{m} \prod_{i=0}^{m-1}\left(n_{0}+i\right)}{\prod_{i=0}^{m-1}\left(s+J\left(n_{0}+i\right)\right)}=\frac{1}{\prod_{i=0}^{m-1}\left(1+\frac{s}{J\left(n_{0}+i\right)}\right)}
$$

It can be shown that the $i$-th moment of this probability density is given by the following expression:

$$
T_{n_{0} \rightarrow n_{0}+m}^{i}=\int_{0}^{\infty} t^{i} \phi_{n_{0}+m}(t) d t=(-1)^{i} \frac{\partial^{i-1} \widetilde{\phi}_{n_{0}+m}(s)}{\partial s^{i-1}}
$$

Thus, Taylor expansion of this probability yields all of its moments, including the mean first-passage time (the first moment):

$$
\widetilde{\phi}_{n_{0}+m}(s)=1-s T_{n_{0} \rightarrow n_{0}+m}+s^{2} T_{n_{0} \rightarrow n_{0}+m}^{2}+\ldots
$$

Thus mean-first passage time is given by:

$$
T_{n_{0} \rightarrow n_{0}+m}=\frac{1}{J} \sum_{j=0}^{m-1} \frac{1}{n_{0}+j}
$$

Taking $m=l$ and $J=k+\lambda$, we recover first-passage time in eqn S13:

$$
T_{n_{0} \rightarrow n_{0}+m}=\frac{1}{k+\lambda} \sum_{j=0}^{l-1} \frac{1}{n_{0}+j}
$$

Similar calculations yields the second moment:

$$
T_{n_{0} \rightarrow n_{0}+m}^{2}=\frac{1}{(k+\lambda)^{2}} \sum_{j=0}^{l} \frac{1}{\left(n_{0}+l-j\right)^{2}}+\frac{1}{(k+\lambda)^{2}}\left(\sum_{j=0}^{l} \frac{1}{\left(n_{0}+l-j\right)}\right)^{2}
$$

And the variance is given by,

$$
\delta T_{n_{0}}=\sqrt{T_{n_{0} \rightarrow n_{0}+m}^{2}-\left(T_{n_{0} \rightarrow n_{0}+m}\right)^{2}}=\frac{1}{(k+\lambda)} \sqrt{\sum_{j=0}^{l} \frac{1}{\left(n_{0}+l-j\right)^{2}}} .
$$

